Itinéraires Itinéraires

Littérature, textes, cultures

2017-2 | 2018

Féminismes quatrième génération

\title{
Les mondes possibles des féminismes contemporains
}

Présentation

Stéphanie Pahud et Marie-Anne Paveau

\section{OpenEdition}

\section{Journals}

Édition électronique

URL : http://journals.openedition.org/itineraires/3785

DOI : 10.4000/itineraires.3785

ISSN : 2427-920X

\section{Éditeur}

Pléiade

\section{Référence électronique}

Stéphanie Pahud et Marie-Anne Paveau, "Les mondes possibles des féminismes contemporains », Itinéraires [En ligne], 2017-2 | 2018, mis en ligne le 10 mars 2018, consulté le 24 septembre 2020. URL http://journals.openedition.org/itineraires/3785 ; DOI : https://doi.org/10.4000/itineraires.3785

Ce document a été généré automatiquement le 24 septembre 2020.

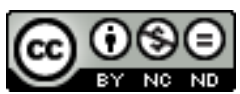

Itinéraires est mis à disposition selon les termes de la licence Creative Commons Attribution - Pas d'Utilisation Commerciale - Pas de Modification 4.0 International. 


\section{Les mondes possibles des féminismes contemporains}

Présentation

Stéphanie Pahud et Marie-Anne Paveau

Quels sont les prochains défis du féminisme? Participer d'une société composée d'individus

libérés de la binarité femmes-hommes qui continue à nous épingler dans des singularités

discriminantes. Le dilemme, c'est que pour déconstruire ces discriminations il faut pouvoir

en faire le diagnostic et mettre en place des actions positives pour y remédier. Il faut donctout au moins dans un premier temps conserver les catégories de la domination tout en ayant pour horizon de les dépasser. Cet au-delà de la complémentarité binaire est un défi exigeant, éminemment politique et complexe. Il est pour moi la condition d'une égalité sans conditions pour tou.te.s et chacun.e. Réjane Sénac, Les Inrocks 2, « Génération féministe », juin 2017, p. 9. Il reste encore un travail important à faire sur la

façon dont les pratiques des locutrices et des locuteurs construisent et / ou déconstruisent le(s) genre(s), les sexualités et les rapports de domination en mobilisant une pluralité de ressources sémiotiques telles que la parole, les textes, les inscriptions graphiques, les images, les conduites vocales, gestuelles, posturales, visuelles, etc. 
Luca Greco, « Présentation », Langage \& Société, n 148, juin 2014, p. 7.

1 Ce numéro d'Itinéraires a pour but de montrer qu'une nouvelle sémiotique féministe (au sens large de production de signes, textes, discours, slogans, symboles, etc. ${ }^{1}$ ) émerge en ce début de $\mathrm{XXI}^{\mathrm{e}}$ siècle, issue des contextes sociaux et politiques contemporains ainsi que des nouvelles donnes, en particulier internationales et intersectionnelles, du militantisme féministe. Cette nouvelle sémiotique féministe, qui diversifie les espaces de déstabilisation de la binarité des sexes et du sens de ces derniers, est emblématique de la lutte contemporaine, notamment décrite par Christine Delphy, comme une « autoémancipation où les opprimé-e-s non seulement luttent pour leur libération, mais la définissent », une lutte « quasi charnelle » (Delphy $2016: 86-87$ ).

2 Outre la succession des générations qui renouvelle naturellement et démographiquement les militant·ess et leurs modes de militance, deux grands facteurs motivent ces innovations sémiotiques-textuelles :

- le développement et l'installation des études de genre, qui bousculent certaines des notions forgées par les féministes de deuxième et troisième génération des années 1960 aux années 1990 : dans la quatrième génération qui produit actuellement des discours militants (militant·es née's autour des années 1980-1990 et/ou prenant la parole à partir des années 2000), la notion de genre implique de penser les rapports de sexe, la sexualité, l'identité, l'oppression, sous un nouvel éclairage, ce qui modifie les productions sémiotiques (Dorlin 2008, Quemener 2012). La question LGBTQIA ${ }^{2}$, la théorie queer, la reconnaissance des minorités racialisées (afroféminisme notamment) modifient les formes et les frontières du féminisme dont le sujet, désormais, comme le déclare Judith Butler dans Trouble dans le genre « n'est pas la défense des femmes » (Butler [1990] 2005), mais celle de toutes les personnes opprimées.

- le discours féministe a en effet étendu son premier objet, la défense des femmes et la promotion de l'égalité de sexes, à un objet plus large et hétérogène: par le biais de l'intersectionnalité, les élaborations sémiotiques-textuelles tiennent compte des autres critères d'oppression et de minorisation, comme la classe, la race ${ }^{3}$ ou la sexualité (Dorlin, dir. 2009, Hill Collins 1990, hooks 1999). On a donc affaire à une utilisation presque hyperonymique du terme féminisme, correspondant dans le fait à la défense des minorités opprimées pour leur sexualité et/ou racialisées, y compris d'ailleurs l'oppression des hommes, saisie à partir des dressages de genre producteurs de l'identité masculine (Pahud 2011).

\section{Traits sémiotiques-textuels des féminismes de quatrième génération}

3 Les féminismes de quatrième génération mobilisent une pluralité de ressources sémiotiques-textuelles et possèdent des traits spécifiques et parfois innovants par rapport aux traditions antérieures. Quatre d'entre eux sont traités dans ce numéro.

\section{La réflexivité}

4 Les féministes de quatrième génération, parce qu'ileelle-s sont pris·es dans le réseau d'oppressions dessiné par l'intersectionnalité, intègrent le plus souvent à leurs prises de parole une description de soi et procèdent à un exercice de "désidentification »: 
déclaration de privilèges de la «femme blanche hétérosexuelle bourgeoise » ou du " mâle blanc hétérosexuel ", selon des expressions désormais stéréotypées (voir par exemple le pamphlet de Félix Boggio Éwanjé-Épée et Stella Magliani-Belkacem, Les Féministes blanches et l'Empire, publié en 2012 à La Fabrique), ou au contraire déclaration d'oppression de la part, par exemple, de la femme noire lesbienne qui sera ségréguée à la fois pour son genre, sa couleur et sa sexualité, ou de l'homme trans' arabe marginalisé à la fois pour son changement de genre/sexe et son appartenance ethnico-culturelle. D'une manière plus générale, les textes-signes féministes de quatrième génération possèdent souvent une dimension métalangagière ou métadiscursive, abordant fréquemment des points de langage ou d'expression, dénonçant des emplois sexistes, proposant des formes lexicalement respectueuses, etc.

\section{La mobilisation du corps}

5 L'irruption d'un activisme féministe dénudé (Femen, Slutwalks), mais également l'émergence de nombreux projets artistiques ou photographiques présentant le corps féminin sous le signe de la libération ou encore de phénomènes ponctuels obligent à poser la question du corps comme dispositif sémiotique de militance et de résistance (Bard 2014). La méthode n'est pas nouvelle et la "manufestation", selon un terme québécois, a évidemment précédé les féministes de quatrième génération (Guillon 2008), mais la banalisation du procédé dans les luttes féministes contemporaines mérite un examen et un questionnement (Fraisse 2014, Paveau 2014). Au-delà du dénudement en public, le corps des femmes constitue le siège d'un discours militant, comme le montre un exemple parmi d'autres: la protestation des femmes turques par la publication sur Twitter notamment de photos d'elles en train de sourire ou de rire aux éclats en août 2014, après que le Premier ministre Bulent Arinç a déclaré : « Une femme doit conserver une droiture morale, elle ne doit pas rire fort en public. »

\section{L'exploitation des univers numériques}

6 La mobilisation de la blogosphère et des réseaux sociaux numériques (Twitter, Facebook notamment, mais aussi des réseaux de partage de vidéos comme YouTube, ou d'images fixes ou animées comme Tumblr) confère aux productions textuellessémiotiques féministes des dimensions d'abondance et de rapidité inédites (viralité des publications sur le Web 2.0). Les possibilités sémiotiques offertes par les plateformes de publication en ligne permettent en outre la création de nouvelles formes de discours militant, composites technodiscursifs, verbo-iconiques, fixes ou animés (avatars, mèmes, pancartes, cartoons) qui situent la production militante dans une plurisémioticité dynamique (Paveau 2017). La notion de «mansplaining», par exemple, création récente des féministes anglophones transférée telle quelle en France (la traduction proposée, "mecsplication ", ne rencontre guère de succès), fait l'objet de nombreuses réalisations graphiques ayant finalement produit un véritablement mème, comme le montre l'illustration ci-dessous. 
Les créations graphiques autour du mansplaining (page 1 de la recherche Google images « mansplaining»)
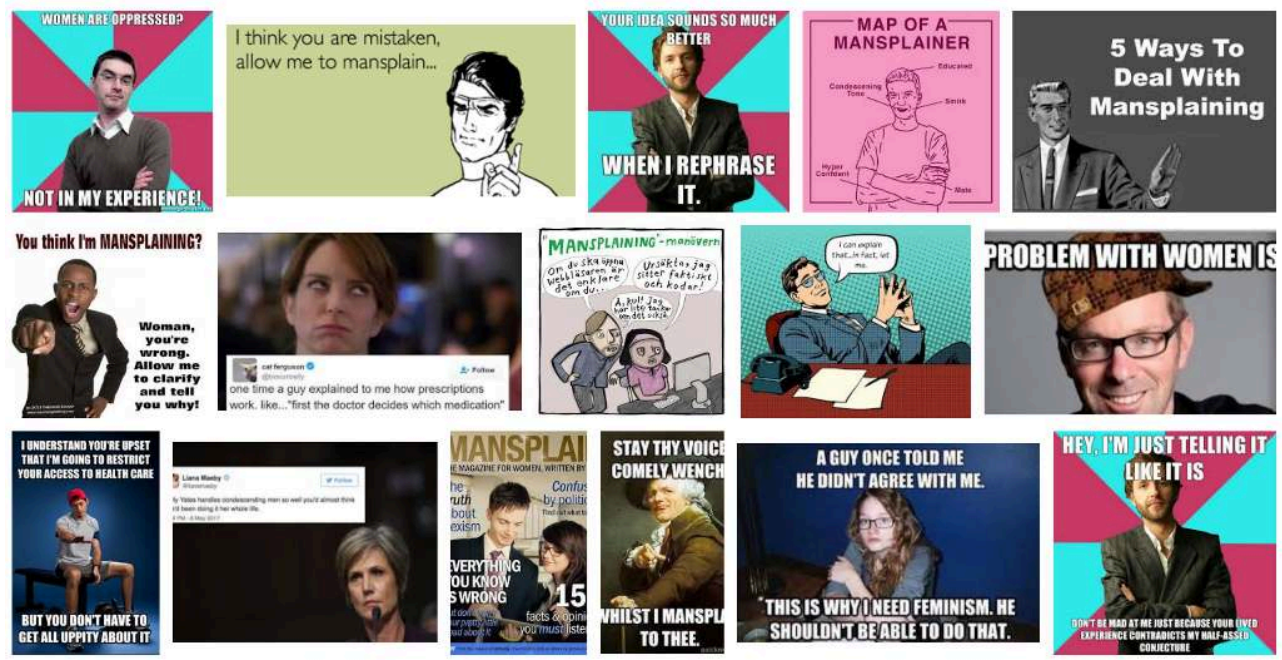

\section{Le dialogue avec l'antiféminisme}

7 Le féminisme de quatrième génération intègre un dialogue avec les antiféminismes qui se construisent en symétrie. L'irruption d'une part d'un activisme féministe masculin et de mouvements masculinistes antiféministes et d'autre part de mouvements féministes antagonistes, invite à poser la question de l'incidence des discours et imaginaires antiféministes dans l'émergence d'une nouvelle sémiotique féministe, mais aussi, symétriquement, de l'incidence de cette nouvelle sémiotique féministe dans le renforcement de postures antiféministes.

\section{Composition du numéro}

8 Le numéro accueille des textes émanant de disciplines diverses des lettres, sciences humaines et sociales, tous les textes se fédérant autour du point de rassemblement que constitue l'objet «productions textuelles-sémiotiques». Sont représentées la littérature, l'histoire, les études hispaniques, les sciences de l'information et de la communication, la linguistique textuelle, la sociologie, la philosophie politique. L'aire culturelle et linguistique concernée est essentiellement la France, avec des ouvertures vers les Amériques, Brésil, Québec et États-Unis.

Les auteures du numéro sont par ailleurs toutes à des degrés divers, féministes. Ce travail s'inscrit en effet dans une épistémologie du point de vue (standpoint epistemology) ne séparant pas discours scientifique et position politique, et, au-delà, réclamant que l'investigation de recherche soit soutenue par un point de vue politique, sans lequel elle serait invalide (Harding 2004). Le numéro présente une approche scientifique intégrant à la procédure d'analyse la nécessité des dimensions subjectives de ses auteur·es, et transposant de la vie à la recherche la célèbre notation de Puig de la Bellacasa : «Les conditions de vie sont aussi des conditions de vue » (Puig de la Bellacasa 2013).

Les trois premiers articles proposent des cadrages d'ordre historique et épistémologique. L'article de Bibia Pavard, "Faire naître et mourir les vagues: comment s'écrit l'histoire des féminismes" fait d'abord un bilan des usages 
académiques et militants de la métaphore des "vagues féministes» afin de lui redonner une historicité et de montrer le poids des enjeux politiques dans son utilisation. À travers le cas de la France, elle montre comment cette métaphore témoigne de la circulation des appellations entre le discours militant et le discours académique et révèle les tensions existantes pour savoir qui décide de faire naître et mourir les vagues.

11 Dans sa contribution, "Des Femmes, des sciences et des technologies: une analyse textuelle de Bitch 2000-2016", Heidi Gautschi prend pour point de départ le débat récurrent sur la disparité des genres dans les métiers scientifiques et techniques ainsi que le débat plus fondamental sur la définition de la science. Dans le but de comprendre comment une publication féministe se retrouve dans ce débat, elle entreprend une analyse textuelle d'une sélection d'articles qui ont pour sujets principaux la technologie, ou la science apparus dans Bitch: A Feminist Response to Pop Culture entre 2000 et 2016.

12 La contribution de Stéphanie Pahud, "L'électoralisation du féminisme: un nouvel antiféminisme ordinaire ", s'intéresse aux rapports au féminisme. Elle pose l'hypothèse que l'on assiste en ce début de $\mathrm{xxI}^{\mathrm{e}}$ siècle à l'« électoralisation » de ce dernier : valeurs et principes féministes sont désormais plus ou moins "cotés ", et l'opposition entre féminisme et anti-féminisme est assourdie par l'opposition entre féminisme «à la mode» et «féminisme has been ». Par le biais d'une analyse discursive de quelques positionnements féministes et antiféministes, elle fait dialoguer théorie du discours, linguistique textuelle et études de genre pour montrer que le féminisme est un acte de création à problématiser et que les appropriations complexes qu'il produit gagnent à être repolitisées.

13 Les questions du corps et des sexualités fédèrent les contributions suivantes. L'article d'Anne-Charlotte Millepied, «Le pouvoir des mots et des corps. L'autodéfense féministe, lieu de production de scripts sexuels alternatifs", interroge le potentiel subversif de la pratique de l'autodéfense féministe, qui agit précisément tant au niveau du discours et de la représentation qu'à celui de l'expérience vécue. À travers l'analyse des différents éléments qui sous-tendent l'imbrication du langage et du corps dans un stage d'autodéfense féministe, l'auteure montre dans quelle mesure cette pratique peut constituer un lieu de production de scripts sexuels alternatifs.

La contribution d'Isabelle Boisclair, "Solange te parle féminisme. Sexe, genre et sexualité dans les capsules de Solange te parle » analyse en regard du discours et de la représentation du sexe, du genre et de la sexualité cinq capsules du blog Solange te parle, fondé en 2011 par Ina Mihalache, née à Montréal en 1985 d'un père roumain et d'une mère québécoise et installée en France en 2004. L'auteure pose l'hypothèse qu'en offrant des textes qui questionnent les normes sociales du féminin, du masculin et des rencontres sexuelles et en se mettant en scène sans apparat, Mihalache décape les modèles dominants, promouvant des subjectivités et des sexualités plus brutes - plus authentiques - recadrant ainsi de nouveaux possibles identitaires.

Les nouveaux discours féministes étant généralement le fait de féministes jeunes, militantes et développant des sémiotiques particulières, ce numéro donne la parole, sous la forme d'une interview réalisée par Stéphanie Pahud, à une jeune militante. Coline de Senarclens, née en Suisse en 1985, se définit elle-même comme "féministe, gauchiste, antiraciste ». Elle est régulièrement sollicitée par les médias et s'investit dans la lutte pour l'autodétermination des femmes. Cofondatrice de La Marche des 
Salopes Suisse - collectif né en 2012, devenu une association en $2014^{5}$-, elle est par ailleurs l'auteure de Salope!, un essai paru en 2014 chez Hélice Hélas Éditeur, dans lequel elle décrit la stigmatisation sexuelle dont elle a été victime adolescente et la manière dont elle en a opéré la resignification en devenant militante.

Les deux derniers articles de cette livraison rendent compte de l'un des traits majeurs des féminismes contemporains, leur formulation en ligne. L'article de Thérèse Courau, "Parole de queerzine : archive_corps_technologie », analyse quelques traits et enjeux des nouvelles sémiotiques post-féministes liées au développement des blogzines queer sur le Web 2.0 qui fonctionnent depuis quelques années comme de puissantes contretechnologies du genre dans la production de nouvelles subjectivités post-identitaires. Il prend pour corpus le queerzine Parole de Queer, originellement distribué en version papier dans les grandes villes catalanes comme Barcelone ou Valence mais surtout largement relayé et enrichi par la diffusion numérique, grâce au blog et aux réseaux sociaux associés au zine. Il analyse la manière dont ce média fonctionne comme une interface parole_corps_technologie qui sert la politique queer de prolifération virale de représentations inédites des genres, des sexualités et des corps post-organiques, laquelle joue de la fluidité et de la plurisémioticité des univers numériques.

Dans sa contribution, «Safe space et charte de langage, entre subversion et institution d'une Constitution", Anne Plaignaud part du constat que l'une des querelles générationnelles dans le féminisme porte sur le statut du sujet de la lutte. En prenant pour exemple un groupe Facebook luttant contre le harcèlement, l'auteure explore une double hypothèse : elle tente de voir si ce n'est pas un décalage vers la notion de public qui est en train de s'amorcer à travers de simples « récits de soi " postés jour après jour, et si la fondation de ce public ne vient pas d'une coercition a priori de l'espace discursif.

S'ajoute enfin à ce numéro d'Itinéraires un excursus transatlantique dans une somme de deux volumes publiés en 2017 par le groupe de recherches brésilien « Mulheres em Discurso, lugares de enunciaçao e processos de subjectivaçao » (MULHERDIS) consacrés à la compréhension des modes d'inscription des identités de genre dans la construction discursive des sujets.

\section{BIBLIOGRAPHIE}

Bard, Christine, 2014, " "Mon corps est une arme”, des suffragettes aux Femen », Les temps modernes, $\mathrm{n}^{\circ} 678$, p. 213-240.

Boggio, Éwanjé-Épée Félix et Magliani-Belkacem, Stella, 2012, Les Féministes blanches et l'Empire, Paris, La Fabrique.

Butler, Judith, [1990] 2005, Trouble dans le genre, trad. C. Kraus, Paris, La Découverte.

Delphy, Christine, 2016, « Plus fortes entre elles », Le Monde diplomatique. Manière de voir, $\mathrm{n}^{\circ} 150$, «Femmes. La guerre la plus longue », p. 86-87.

Dorlin, Elsa, 2008, Sexe, genre et sexualités. Introduction à la théorie féministe, Paris, PUF. 
Dorlin, Elsa (dir.), 2009, Sexe, race, classe, Paris, PUF.

Fraisse, Geneviève, 2014, Les Excès du genre, Paris, Lignes.

Guillon, Claude, 2008, Je chante le corps critique : les usages politiques du corps, Paris, H\&O.

Harding, Sandra (dir.), 2004, The Feminist Standpoint Theory Reader. Intellectual \& Political Controversies, Londres, Routledge.

Hill Collins, Patricia, 1990, Black Feminist Thought: Knowledge, Consciousness and the Politics of Empowerment, New York, Londres, Routledge.

hooks, bell, 1999, Ain't I a Woman: Black Women and Feminism, Boston, South End Press.

Pahud, Stéphanie, 2011, Petit traité de désobéissance féministe, Neuchâtel, Arttesia Éditions.

Paveau, Marie-Anne, 2014, «Quand les corps s'écrivent. Discours de femmes à l'ère du numérique ", dans É. Bidaud (dir.), Recherches de visages. Une approche psychanalytique, Paris, Hermann, p. 207-241.

Paveau, Marie-Anne, 2017, « Féminismes 2.0. Usages technodiscursifs de la génération connectée ", Argumentation et Analyse du Discours, n 18, [En ligne], https:// journals.openedition.org/aad/2345.

DOI : $10.4000 /$ aad.2345

Puig de la Bellacasa, Maria, 2013, Politiques féministes et construction des savoirs. «Penser nous devons »!, Paris, L’Harmattan.

Quenemer, Nelly, 2012, « Les contradictions corps/langage comme moteur du rire. Parodies et incarnations de genre chez les humoristes femmes en France », dans L. Greco et N. Chetcuti (dir.), La face cachée du genre : le rôle du langage dans la transmission et la contestation des normes, Paris, Presses Sorbonne Nouvelle, p. 85-103.

\section{Documentation du numéro}

\section{N.B. : Tous les liens ont été vérifiés le 30 novembre 2017.}

Ali, Zahra, 2012, Féminismes islamiques, Paris, La Fabrique.

Bard, Christine, 1995, Les Filles de Marianne. Histoire des féminismes 1914-1940, Paris, Fayard.

Baril, Alexandre, 2015, « Sexe et genre sous le bistouri (analytique) : interprétations féministes des transidentités ", Recherches féministes, vol. 28, nº 2, p. 121-141.

Blais, Mélissa et Dupuis-Déri, Francis, 2014, « Antiféminisme : pas d'exception française », Travail, genre et sociétés, $\mathrm{n}^{\circ} 32$, p. 151-156.

Bourcier, Marie-Hélène et Moliner, Alice, 2012, Comprendre le féminisme, Paris, Max Milo.

Dubesset, Mathilde, 2000, Des femmes en mouvement, catholiques et protestantes au XXe siècle, Grenoble, Cahiers de Meylan.

Dupuis-Déri, Francis, 2004, « Féminisme au masculin et contre-attaque "masculiniste" au Québec », Mouvements, $\mathrm{n}^{\circ}$ 31, p. 70-74.

Dupuis-Déri, Francis et Lamoureux, Diane (dir.), 2015, Les antiféminismes : analyse d'un discours réactionnaire, Montréal, Les Éditions du Remue-ménage. 
Greco, Luca, 2014, « Mise en scène d'une transition scientifique et identitaire : expérience narrative, réflexivité et catégorisation », Semen, $n^{\circ} 37$, [En ligne], https:// journals.openedition.org/semen/10204.

Greco, Luca (dir.), 2014, Langage et société, $\mathrm{n}^{\circ}$ 148, « Recherches linguistiques sur le genre : bilan et perspectives ».

Greco, Luca, 2014, « Les recherches linguistiques sur le genre : un état de l'art », Langage et société, $\mathrm{n}^{\circ} 148$, p. 11-29.

Greco, Luca (dir.), 2015, Langage et société, nº 152, « Genre, langage et sexualité : données empiriques ».

Greco, Luca, 2017, « La performance au carrefour des arts et des sciences sociales : quelles questions pour la sociolinguistique? », Langage et société, $n^{\circ}$ 160-161, p. 301-317.

Greco, Luca et Mondada, Lorenza, 2014, «Identités-en-interaction : vers une approche multidimensionnelle », dans L. Greco, L. Mondada et P. Renaud (dir.), Identités en interaction, Limoges, Lambert-Lucas, p. 1-26.

Groult, Benoîte, 2010, Le Féminisme au masculin, Paris, Grasset.

Harding, Sandra, 1993, « Rethinking Standpoint Epistemology: What is Strong Objectivity? », dans L. Alcoff et E. Potter (dir.), Feminist Epistemologies, New York, Londres, Routledge.

Las, Nelly, 2011, Voix juives dans le féminisme. Résonances françaises et anglo-américaines, Paris, Champion.

Latte, Abdallah Stéphanie, 2010, «Les féminismes islamiques au tournant du $\mathrm{XxI}^{\mathrm{e}}$ siècle ", Revue des mondes musulmans et de la Méditerranée, $\mathrm{n}^{\circ}$ 128, [En ligne], https://journals.openedition.org/ remmm/6822.

Riot-Sarcey, Michèle, 2008, Histoire du féminisme, Paris, La Découverte, coll. « Repères ».

Zoppi Fontana, Monica et Ferrari, Ana Josefina (dir.), 2017, Mulheres em Discurso : gênero, linguagem e ideologica, volume 1, Campinas, Pontes.

Zoppi Fontana Monica et Ferrari, Ana Josefina (dir.), 2017, Mulheres em Discurso : identificaçoes de gênero et praticas de resistencia, volume 2, Campinas, Pontes.

\section{NOTES}

1. Pour aller vite, nous employons le terme composé textuel-sémiotique pour désigner cet ensemble.

2. Pour « Lesbienne, gay, bi·e, trans', queer, intersexe » (le sigle peut également être augmenté du P de pansexuel, LGBTQIAP).

3. Nous assumons l'emploi du terme race à la manière anglophone, et conformément à de nombreux usages à la fois scientifiques et militants en France : la race est considérée ici comme un marqueur social dans le cadre d'une réflexion sur l'oppression comme système. En ce sens, être noiree, arabe ou blanche, c'est être pris·e dans un système d'oppression, du côté de l'oppriméee ou de l'oppresseur'e, système hérité des racialisations officiellement mises place par les colonialismes.

4. Même remarque sur arabe que pour l'emploi de race.

5. https://www.facebook.com/slutwalksuisse/. 


\section{AUTEURS}

\section{STÉPHANIE PAHUD}

Université de Lausanne, École de français langue étrangère (EFLE)

MARIE-ANNE PAVEAU

Université Paris 13, Pléiade (EA 7338) 